\title{
Eccellenza assistenziale e dialisi peritoneale
}

\author{
Alessia Delalio ${ }^{1}$, Maria Pia Zito ${ }^{2}$, Marisa Pegoraro ${ }^{3}$ \\ ${ }^{1}$ Referente nazionale GIPD-EDTNA/ERCA filiale italiana \\ ${ }^{2}$ Referente centro GIPD-EDTNA/ERCA filiale italiana \\ ${ }^{3}$ Presidente EDTNA/ERCA filiale italiana
}

\begin{abstract}
Patients' care excellence and Peritoneal dialysis
Abstract. In parallel to the diversification of the strategies of peritoneal dialysis, a large impulse was given by the search for specific competences in the field of patients' care. A national research named "EDTNA ERCA" (in 2005) allowed us to learn about the different organizational realities and the continuity of welfare-oriented care, which is today a prerequisite for the building of the network between hospital and territory. Therapeutic education and adult-specific technical skills characterized the formation that EDTNA/ERCA has proposed, but these do not support the thesis of the marginality of the technique and the skills that its practice requires. This last aspect can be one of the critical issues for its development.
\end{abstract}

Key words: Peritoneal dialysis, Nursing, Training, Competences, Health-care management

Conflict of interest: None.

Financial support: None.

Accettato: 21 Dicembre 2013

La sua elevata e indubbia preparazione scientifica ... grande capacità riflessiva ... calma interiore ... buon senso non comune ... capacità di essere umile e una naturale empatia, che, associate alla facilità di entusiasmarsi, ... lo rendevano ...

(Dr. Marco Lombardi)

... "Amedeo De Vecchi: un uomo, medico e nefrologo"

A distanza di dodici anni dall'articolo di Amedeo De Vecchi, parallelamente al diversificarsi delle strategie di dialisi peritoneale, ha avuto un grosso impulso la ricerca di competenze assistenziali specifiche che potessero far emergere questa metodica dalla marginalità in cui i nostri stessi colleghi, medici e infermieri, la relegano.

La definizione del termine "marginale" lega e sé due paradigmi "ciò che non ha peso" e "ciò che non ha valore" (1), due elementi che, in dialisi peritoneale, convivono contrapposti. Il concetto di "peso" della metodica è ampiamente rappresentato dai dati di penetrazione della stessa, che, rispetto alla totalità dei trattamenti dialitici, ce la mostrano come una metodica di "poco peso".

Il "valore" di questa metodica, dal punto di vista clinico, è ampiamente dimostrato ma, unitamente alla clinica, l'assistenza che i pazienti ricevono in dialisi peritoneale rappresenta un'eccellenza nel contesto della professione infermieristica.

L'assistenza infermieristica preventiva, curativa, riabilitativa e palliativa, di natura tecnica, relazionale ed educativa trova ampi contesti di sviluppo in dialisi peritoneale.
EDTNA/ERCA, in questi anni, si è fatta promotrice di una ricerca nazionale "sulla ratio infermiere/n. pazienti nei servizi di dialisi peritoneale in Italia: risultati, trend e loro utilizzo" che ha permesso, nel 2005, di conoscere le diverse realtà organizzative dei centri italiani che praticano la dialisi peritoneale e le funzioni delle diverse figure professionali che compongono il gruppo multidisciplinare, con particolare riferimento al ruolo dell'infermiere.

Gli ambiti in cui gli infermieri di DP operano sono estremamente diversificati: dall'ambulatorio alla degenza, al domicilio e a contesti residenziali sociali, confermando la capacità di adattare la propria professionalità al bisogno di salute. L'attività assistenziale vede come primo protagonista il paziente, ma non unico soggetto di interventi che, nel processo educativo di gestione della patologia, vedono anche il coinvolgimento della famiglia e di colleghi di altre realtà operative in cui sono ricoverati i pazienti.

L'assistenza al paziente dializzato peritoneale oggi si fonda sulla continuità di un processo assistenziale che inizia nella fase pre dialitica, prosegue nell'addestramento alla metodica e alla gestione della malattia, seguendo la persona anche al suo domicilio, e, infine, passando attraverso il confronto con eventuali complicanze, accompagna la persona, ove possibile, al trapianto e, ove non è possibile, alla morte.

L'obiettivo dell'empowerment del paziente rappresenta, in dialisi peritoneale, un elemento consolidato, supportato dai dati relativi al ridotto numero di peritoniti, un indicatore di 
processo fortemente rappresentativo della qualità dell'assistenza. Unitamente alla definizione di una specializzazione professionale, EDTNA/ERCA ha analizzato l'ambito delle politiche sanitarie (Milano 2010-Bari 2011), mettendo a confronto due sistemi sanitari regionali fortemente orientati alla domiciliazione delle cure, come obiettivo strategico cogente per la qualità di vita della persona e la sostenibilità economica delle cure.

La continuità della cura presente in dialisi peritoneale è il presupposto indispensabile per la costruzione della rete tra ospedale e territorio, ma la sua realizzazione richiederà un ulteriore sforzo nello sviluppo di competenze di management sanitario. Educazione terapeutica e competenze tecniche educative specifiche per gli interventi rivolti agli adulti hanno caratterizzato i percorsi formativi che EDTNA/ERCA ha proposto ai colleghi infermieri e medici, spesso ospiti delle nostre giornate formative in un percorso di professionalizzazione e presa di coscienza, che sicuramente non supportano la tesi della marginalità della metodica e delle competenze professionali che la sua pratica richiede.

Proprio quest'ultimo aspetto può essere la criticità per il suo sviluppo: non tutti i professionisti hanno avuto modo di sviluppare le competenze professionali necessarie per sostenere la pratica della metodica peritoneale.

La dialisi peritoneale non è un impegno marginale per i professionisti che decidono di promuoverla nei propri centri.

In questi anni gli infermieri, nei propri contesti lavorativi, hanno lavorato accanto alla professione medica per costruire il "valore salute" ritenendo che questo sia uno dei maggiori valori a cui una società moderna possa ambire per i propri cittadini/pazienti.

Le aree di sviluppo futuro per gli infermieri sono orientate alla produzione di Linee Guida che sostengano la pratica professionale, che non si esaurisce nel "garantire la corretta applicazione delle prescrizioni terapeutiche e diagnostiche" (D.L. 739/94) e nella definizione del "core curriculum" professionale dell'infermiere di dialisi peritoneale, a sostegno del valore di una specializzazione.

La definizione di outcome in dialisi peritoneale e la loro correlazione con il modello organizzativo rappresentano la strategia vincente per sostenere il valore di una dialisi, spesso emarginata dalla stessa nefrologia, che oggi rischia di essere appiattita da un modello di "care" orientato all'intensità della cura e non alla specificità dei "bisogni della persona".

\section{Riassunto}

Parallelamente al diversificarsi delle strategie di dialisi peritoneale, un grosso impulso ha avuto la ricerca di competenze assistenziali specifiche. Una ricerca nazionale EDTNA/ ERCA (2005) ha permesso di conoscere le diverse realtà organizzative e assistenziali orientate alla continuità delle cure, oggi presupposto indispensabile per la costruzione della rete tra ospedale e territorio. Educazione terapeutica e competenze tecniche educative specifiche per gli adulti hanno caratterizzato la formazione che EDTNA/ERCA ha proposto, non supportando la tesi della marginalità della metodica e delle competenze professionali che la sua pratica richiede. Proprio quest'ultimo aspetto può essere una delle criticità per il suo sviluppo.

Parole chiave: Dialisi peritoneale, Infermieristica, Formazione, Competenze, Management sanitario

Dichiarazione di conflitto di interessi: Gli Autori dichiarano di non avere conflitto di interessi.

Contributi economici degli autori: Gli Autori dichiarano di non aver ricevuto sponsorizzazioni economiche per la preparazione dell'articolo.

\section{Bibliografia}

1. Enciclopedia Treccani.

\section{Letture consigliate}

- $\quad$ Decreto ministeriale 739/94 profilo dell'infermiere.

- Ricerca EDTNA/ERCA sulla ratio infermiere/n. pazienti nei servizi di dialisi peritoneale in Italia: risultati, trend e loro utilizzo (2005).

- Consensus conference: La Dialisi Peritoneale quale protagonista nel processo di deospedalizzazione e della gestione assistenziale per intensità di cure. Milano 2010-Bari 2011. 